Auch nach der Aufarbeitung eines großen Puppenund Fliegenmaterials trat niemals eine Substanz auf, welche die für die Kynurensäure typischen Eigenschaften besaß. Der Abbau des Kynurenins muß hier also in anderer Richtung verlaufen, was angesichts der Erbformel sehr auffällig ist.

III. Oberhalb des Fleckes 2 (Kynurensäure) liegt bei $R_{\mathrm{f}}=0,8$ noch ein weiterer Fleck 1 , den wir aber vorläufig weder identifizieren noch seinem Vorkommen nach in die Ommochrombildung einordnen konnten und der deshalb hier nur der Vollständigkeit halber erwähnt werden soll. Er ist bei allen untersuchten Rassen vorhanden, und zwar in den Puppen und in den Fliegen. Der Stoff fluoresziert blau-violett, ist ninhydrin-negativ, färbt sich mit Methylrot rosa-rot, mit $p$-Dimethylaminobenzaldehyd braun an und reagiert nicht mit Äthyl- $\alpha$-Naphtylamin, ist also nicht mit Anthranilsäure identisch, die den $R_{\mathrm{f}}$-Wert 0,7 besitzt.

IV. Unterhalb des Fleckes 3 (Kynurenin) folgen zwei weitere fluoreszierende Flecken 4 und 5 mit den $R_{\mathrm{f}}$-Werten 0,45 und 0,37 (Abb. 3), die offenbar mit der Bildung des roten Augenfarbstoffes zusammenhängen, weil sie bei bw-, cnbw- und vbw-Tieren fehlen. Diese beiden Substanzen interessieren uns natürlich besonders und sind Gegenstand weiterer Untersuchungen. Ein noch weiter unten liegender gelb fluoreszierender Fleck $7^{* *}$ mit dem $R_{\mathrm{f}}$-Wert 0,21 kommt nur bei Fliegen vor, die braunes Augenpigment zu bilden vermögen ( + und bw). Dann folgt ein violett fluoreszierender Fleck $8\left(R_{\mathrm{f}}=0,15\right)$, dessen Farbe bei den Puppen, die rotes Augenpigment bilden $(+, \mathrm{cn}, \mathrm{v})$ gegen Ende der Verpuppungszeit in Blau umschlägt, bei den braun- und weißäugigen Puppen (bw, cn bw, vbw, w) dagegen nicht. Und schließlich finden sich bei der von uns verwendeten Technik in der Nähe des Startpunktes bei $R_{\mathrm{f}}=0,09$ und 0,03 zwei weitere Stoffe, die bereits bei Tageslicht orange gefärbt sind und offensichtlich Komponenten des roten Augenfarbstoffes selbst darstellen (Fleck 9 und 10).

** Der bei $R_{\mathrm{f}}=0,31$ liegende Fleck 6 tritt bei allen untersuchten Drosophilarassen auf, hat mit der Augenfarbe also offenbar nichts zu tun.

\title{
Mitosegifte und Funktions-Formwechsel bei Zellen des Mäuse-Ascitescarcinoms
}

\author{
Von Wilhelm HomanN \\ Aus dem Pathologischen Institut der Universität Erlangen (Direktor: Prof. Dr. Erich Mü ller) \\ (Z. Naturforschg. 9 b, 792-796 [1954]; eingegangen am 29. September 1954)
}

1. Es wird gezeigt, daß es möglich ist, mit den bekannten cytostatischen Substanzen Trypaflavin und Colchicin den Funktions-Formwechsel von Zellen des MAC zu beeinflussen.

2. Einwirkung von Trypaflavin führt zu schweren Veränderungen im Bereich des chromatischen Apparates des Kernes und damit zum Ausfall aller Zeichen einer funktionellen Zellphase. Die Folge dieser Erscheinungen ist die Verhinderung des Eintritts der Zellen in die Phase der Teilung.

3. Nach Colchicin-Einwirkung hingegen tritt die funktionelle Phase der MAC-Zellen verstärkt in Erscheinung. Der Nucleolus wird häufig sehr groß, oft finden sich zwei oder mehr Nucleoli in einem Kern. Es wird angenommen, daß durch die Aktivierung der funktionellen Zellphase die Teilungstendenz der Zellen in gewissem Umfang zunächst gesteigert wird. Die eigentliche spindel-arretierende Wirkung des Colchicins setzt erst später ein.

$\mathrm{N}$ Tach A ltmann ${ }^{1,2}$, Grundmann und Marquardt ${ }^{3}$, Marquardt $t^{4}$ und Grundman ${ }^{5}$ lassen sich im Leben einer Zelle zwei grundsätzlich verschiedene Phasen unterscheiden, von denen eine

1 H. W. Altm a n n, Z. Krebsforsch. 58, 632 [1952].

2 H. W. A l t m a n n, Grundl. u. Praxis chem. Tumorbehandlung, 190 S., Springer 1954.

3 E. Grundmann u. H. M a rqua rdt, Naturwissenschaften 40,557 [1953]. jede durch festumrissene morphologische Merkmale charakterisiert ist. Die eine Phase ist der Vermehrung der Zellen gewidmet und läuft unter den bekannten Zeichen des Teilungs-Formwechsels ab, während die andere der Funktion der Zelle dient und durch den

4 E. Grundmann u. H. M a rqua rd t, Chromosoma 6, 115 [1953].

5 E. Grundmann, Grundlagen u. Praxis chem. Tumorbehandlung, 187 S., Springer 1954. 
Funktions-Formwechsel gekennzeichnet ist, der sich vor allem durch den Vorgang der Kernsekretion erkennen läßt. Zwischen Teilungs-Formwechsel und dem auch als Intermitosephase bezeichneten Funktions-Formwechsel liegt die sog. Interphase, die der Dekondensation der Chromosomen nach der Telophase und ihrer Kondensation vor der anschließenden Prophase dient.

Alle Phasen sind begleitet von Veränderungen des Gehaltes der Kerne an Desoxyribosenucleinsäure (DNS), und zwar dergestalt, daß sich der Gehalt an DNS vom Ausgang der Telophase bis zur nächsten Prophase etwa parallel zur Volumenzunahme der Kerne linear verdoppelt. Mehr oder minder in der Mitte zwischen den Endwerten des DNS-Gehalts der Kerne liegt die durch den Funktions-Formwechsel ausgezeichnete Phase der eigentlichen Arbeitsleistung der Zelle.

Eine große Anfälligkeit des Teilungs-Formwechsels schädigenden Einflüssen gegenüber ist schon lange bekannt und hat u. a. in der Anwendung der sog. cytostatischen Substanzen in der Tumorbehandlung seinen Niederschlag gefunden. Über Eingriffsmöglichkeiten in den Funktions-Formwechsel aber und deren Bedeutung für das nachfolgende Schicksal der Zelle ist bis heute kaum etwas bekannt geworden. Lediglich $\mathrm{M}$ arquardt berichtet darüber, $\mathrm{da}$ ionisierende Strahlen und einige radiomimetische Substanzen an Interphasekernen und, allerdings nur unter erheblicher Steigerung der Dosis, auch in der funktionellen Zellphase wirksam werden können. Eine weitere Klärung der Frage schien uns aber wichtig.

Methodisch gingen wir so vor, daß wir auf Zellen des Mäuse-Ascitescarcinoms (MAC), die für cytologische Untersuchungen besonders geeignet sind, Substanzen einwirken ließen, die völlig verschiedene Wirkungsbereiche umfassen, nämlich Trypaflavin und Colchicin. Von diesen beiden entfaltet Trypaflavin seine Wirkung am „Ruhekern“, während Colchicin als ausgesprochenes Mitosebzw. Spindelgift gilt. Die Dosierung erfolgte nach den wiederholt nachgeprüften und bestätigten Angaben von Brodersen 6 .

\section{Befund}

Die Zellen des MAC zeigen nach TrypaflavinEinwirkung die schon von anderen Autoren beschriebene „Hemmung des Schrittes in die morphologisch faßbaren Stadien der Zellteilung " und nach Colchicingaben eine „Arretierung der Mitosen im Spindelstadium“. Darüber hinaus lassen sich aber bei cyto-

6 H. Brodersen, Strahlentherapie 73, 196 [1943]. logischer Auswertung der Ausstrichpräparate einige bemerkenswerte morphologische Befunde erheben, die den Funktions-Formwechsel der Zellen betreffen.

a) Wirkung der Trypaflavin-Behandlung

Nach Einwirkung von Trypaflavin zeigen die Zellen des MAC eine auffällige Vergröberung der Kernstruktur. Die Fäden des sonst zarten Chromatinnetzes werden unregelmäßig verdickt und dabei unscharf konturiert. Die Chromozentren, die mit kleinen Abschnitten entspiralisierter Chromosomen zu identifizieren sind, vergröbern sich ebenfalls und verlieren dabei ihre scharfe Begrenzung (Abb. $\left.1^{*}\right)$. So erscheinen die Kerne häufig wie von Schollen ausgefüllt und sie erinnern mitunter an Endomitosen, nur ist nie in den scholligen chromatischen Elementen ein Haarspalt als Ausdruck eines Teilungsvorgangs zu sehen. Da mit zunehmender TrypaflavinEinwirkung die anfänglich helle, transparente Interchromatin-Substanz undurchsichtig wird und sich im Feulgen-Azanpräparat dunkel anfärbt, erscheinen die Kerne sehr bald dicht strukturiert. Es drängt sich bei diesen Bildern der Gedanke auf, daß es sich bei diesen dichten Kernen um frühe Stadien der später in einer Häufigkeit bis zu $40 \%$ aller Zellen zu beobachtenden Pyknosen handelt, von denen sie sich nur noch durch einen noch größeren Flüssigkeitsgehalt unterscheiden.

Auffallenderweise fehlt sowohl in den Kernen, die erste Zeichen einer Trypaflavin-Einwirkung in Form einer Vergröberung des Chromatinnetzes zeigen, wie auch in denen mit sehr dichter, fast schon präpyknotischer Struktur, regelmäßig der Nucleolus, obwohl im unbehandelten Tumorascites das Fehlen eines Nucleolus in einem Zellkern zu den Seltenheiten gehört. Selbstverständlich sind beim Fehlen des Nucleolus auch keinerlei Zeichen einer Kernsekretion in den durch Trypaflavin-Einwirkung veränderten Zellen zu sehen, so daß offenbar das Trypaflavin die Ausbildung und den Ablauf eines Funktions-Formwechsels bei den Zellen des MAC völlig unterbindet.

\section{b) Wirkung der Colchicin-Behandlung}

Obwohl Colchicin nach den übereinstimmenden Angaben der Literatur in erster Linie als Spindelgift anzusehen ist, finden sich in jedem Ausstrich colchicin-behandelter MAC-Zellen Veränderungen an den Zellen, die der Interphase oder der Intermitosephase zuzuordnen sind. Sie bestehen bei etwa

\footnotetext{
${ }^{*}$ Abb. 1—3 s. Tafel S. 784 b.
} 
18-20\% der Zellen, ähnlich wie nach TrypaflavinEinwirkung, in einer Vergröberung der chromatischen Kernsubstanz, und zwar in erster Linie durch Vergrößerung der Chromozentren. Diese bleiben dabei aber immer scharf begrenzt, auch dann noch, wenn sie sich mit zunehmender Größe im Zentrum aufhellen. Das aufgehellte Zentrum sieht dann in der Feulgen-Azanfärbung blaßrot aus. Immer ist in den Zellen der Nucleolus erhalten, nicht selten wird er extrem groß, so daß er einen beträchtlichen Teil des Kernbinnenraumes einnimmt (Abb. 2 a). Zwei oder mehr Nucleoli sind nicht selten. Sie sind immer scharf begrenzt und verhalten sich färberisch wie die Nucleoli unbehandelter MAC-Zellen: ihre Wand wird also von glatter oder leicht granulierter feulgenpositiver Substanz gebildet, während der Nucleoleninhalt hell-blaßblau (Feulgen-Azan) oder schwach grün (Feulgen-Lichtgrün) angefärbt ist.

Entsprechend dem Vorhandensein von einem oder mehreren Nucleoli in den Zellen des MAC nach Colchicin-Einwirkung sind alle Zeichen des Funktions-Formwechsels in Form der Kernsekretion vorhanden, wie sie an anderer Stelle beschrieben wurden $\left(\right.$ H o m a n $\left.n^{7}\right)$. Der Vorgang ist häufig sogar so deutlich ausgeprägt, daß man, zusammen mit der Feststellung einer oft extrem ausgeprägten Nucleolusvergrößerung, auf eine durch die Colchicin-Behandlung ausgelöste Kernsekretion schließen kann.

Die oben beschriebene Vergröberung der Chromozentren nach Colchicin-Einwirkung auf die Zellen des MAC bleibt nicht stationär, sondern entwickelt sich zu teilweise recht komplizierten Bildern. Bei etwa $1-3 \%$ aller Zellen vergrößern sich die Chromozentren weiterhin und zeigen nach Erreichen einer gewissen Größe zentrale, rosa gefärbte Aufhellungen. Gleichzeitig beginnt der Kern sich vielfach durchzuschnüren, wobei die Schnürfurchen häufig in der Ebene der von dem oder den noch sichtbaren Nucleolen ausgehenden Sekretionskanälchen verlaufen. Sie bilden sich aber auch unabhängig davon. Das Ergebnis dieser Vorgänge ist eine meist vergrößerte Zelle mit einem ebenfalls vergrößerten Kern, der mit seinen vielfachen Ein- und Durchschnürungen den Eindruck erweckt, als bestünde er aus vielfach ineinandergeschachtelten runden Blasen, deren Wand aus chromatischer Substanz besteht und zwischen denen noch feste Stränge chromatischer Substanz liegen. Offenbar stellen diese Bilder Vor-

7 W. H o m a n n, Z. f. Krebsforsch. 59, 673 [1954].

8 Th. W a g n er-J a u re g g, Hoppe-Seyler's Z. physiol. Chem. 239, 188 [1936]. stufen eines Kernzerfalls dar, der zur Bildung von Zellen mit vielen Kleinkernen führt (Abb. 2 a). Von diesen Kleinkernen zeigt nur ein verschwindend geringer Teil die Struktur normaler MAC-Zellkerne, während die meisten lediglich bläschenförmig sind mit einer feulgen-positiven Wand. Demgemäß erscheint es wenig wahrscheinlich, daß alle Kleinkerne chromosomal vollwertig sind. Daß anschließend an einen Kernzerfall eine scheinbar gesetzlose Cytoplasmateilung einsetzen kann, bei der einige Fragmente ohne Kernanteil bleiben, sei nur der Vollständigkeit halber erwähnt.

Eine besondere Betrachtung verdienen die nach Colchicin-Einwirkung in einer Häufigkeit von etwa $3-4 \%$ aller Zellen in den Ausstrichen zu beobachtenden Pseudoamitosen (Abb. 2 b). Es handelt sich um Zellen, deren Cytoplasmaleib in der Mitte hantelförmig oder nach Art der Plasmabewegung in der Telophase eingeschnürt ist, während der Kern keinerlei Zeichen einer Teilungsbewegung oder eines entsprechenden strukturellen Umbaus erkennen läßt. Insgesamt erwecken die Bilder den Anschein, als mache das Cytoplasma einen Teilungsversuch, an dem aber der Kern keinen Anteil nimmt. Offenbar liegt hier eine Störung im sonst synchronen Ablauf von Plasma- und Kernteilung vor.

Zusammengefaßt führt die Einwirkung von Trypaflavin auf die Zellen des MAC zu schweren Veränderungen im Bereich des chromatischen Kernapparates und damit zum Ausfall aller Zeichen einer funktionellen Zellphase. Die Folge dieser Erscheinungen ist eine Verhinderung des Eintritts der Zelle in die Phase der Teilung.

Nach Colchicin-Einwirkung hingegen tritt die funktionelle Phase an den MAC-Zellen verstärkt in Erscheinung. Der Nucleolus wird häufig sehr groß oder es finden sich zwei oder mehr Nucleoli in einem Kern. Alle Stadien einer Kernsekretion sind zu beobachten. Die Vorbereitungen zur Ausbildung des an die Phase der Funktion anschließenden TeilungsFormwechsels sind sicher nicht behindert.

Nach Wagner-Jauregg ${ }^{8}, \mathrm{McIl}$ wain ${ }^{9}$, Let tré ${ }^{10}$ und anderen ist die Grundsubstanz des Trypaflavins, das Acridin, in der Lage, mit Nucleinsäuren Salze zu bilden. Infolgedessen entfaltet es seine Wirkung auf die Zellen des MAC in den Stadien, in denen nach abgeschlossener Teilung die DNS-Synthese einsetzt, also in der Interphase. Zu diesem Zeitpunkt sind die Chromosomen entspiralisiert und ihre Abschnitte weit

9 G. McIlwain, Biochem. J. 35, 1311 [1941].

10 H. Lettré, Z. f. Krebsforsch. 56, 5 [1949]. 
auseinandergerückt. Da nach $\mathrm{M}$ arquardt ${ }^{11}$ die Reaktion des Trypaflavins mit einzelnen Teilen dieser Chromosomen-Abschnitte erfolgt, nämlich mit denen, an denen sich gerade die Nucleinsäure-Bildung vollzieht, kommt es zu einer weiteren Auflockerung einzelner oder mehrerer Chromoneme innerhalb des Gefüges der Chromosomen. Die dadurch ausgelöste Strukturveränderung kann offenbar zu dem Verlust der normalen Chromosomenfunktion innerhalb des Kernes führen; denn ein Nucleolus, der als spezifische chromosomale Leistung entsteht, wird nicht mehr gebildet, wobei möglicherweise eine blockierende Wirkung des Trypaflavins speziell auf nucleinsäure-bildende Fermente (L e t tré) ${ }^{12}$ eine verstärkende Rolle spielt. Daß unter diesen Voraussetzungen der gestörten DNS-Synthese in der Interphase, der gestörten Strukturen und der - post oder propter hoc - fehlenden funktionellen Zellphase, die ja auch für ein normalerweise nachfolgendes Teilungsgeschehen wichtig ist $\left(\operatorname{Homann}^{7}\right)$, der Eintritt der Zelle in die Mitose unterbleibt, ist verständlich. Die Wirkung des Trypaflavins selbst ist als spezifisch anzusehen.

Wesentlich andere Verhältnisse liegen nach der Einwirkung von Colcichin auf die Zellen des MAC vor, obwohl eine Wirkung, entgegen der bekannten Definition des Colchicins als Spindelgift, auch am noch uneröffneten Kern stattfindet. Auch hierbei kommt es zu einer "Verklumpung“ der chromatischen Kernsubstanz. Sie betrifft aber in erster Linie die Chromozentren, natürlich auch die, welche an der Nucleolenbildung beteiligt sind, und ist ihrem Wesen nach anders geartet als die nach TrypaflavinEinwirkung zu beobachtende. Im Gegensatz zu dieser ist nach Colchicingaben die chromatische Substanz im Bereich der Chromozentren immer scharf begrenzt und stark verdichtet, so daß hier der Eindruck einer Massenzunahme und Kondensation feulgen-positiver chromatischer Substanz entsteht. Da diese über den Rahmen des Normalen hinaus ausgeprägt ist, liegt die Annahme nahe, daß unter Colchicin-Einwirkung bei den Zellen des MAC eine Beschleunigung der DNS-Synthese einsetzt.

Für diese Überlegung spricht der beschriebene Befund einer auffälligen Nucleolus-Vergrößerung nach Colchicin-Einwirkung sowie die ebenfalls häufige Bildung von zwei oder mehr Nucleolen in einem Kern. Diese Erscheinungen, die allgemein als Aus-

11 H. M a rquardt, Naturwissenschaften 40, 557 [1953].

$12 \mathrm{H}$. L e t tré, Grundl. u. Praxis chem. Tumorbeh., 153 S., Springer 1954. druck einer hohen Aktivität von Zellen angesehen werden, entstehen als spezifisch chromosomale Leistung und sind demzufolge im vorliegenden Fall Folge der gesteigerten DNS-Synthese.

Dabei ist an den Zellen mit stark vergrößertem Nucleolus oder mit zwei oder mehreren Nucleoli stets der Vorgang der Kernsekretion, d. h. der Abgabe von im Nucleolus gebildeten Substanzen an das Cytoplasma, eindeutig zu beobachten. Es ist daraus zu folgern, daß bei den Zellen des MAC unter Colchicin-Einwirkung nach der primär einsetzenden verstärkten Kondensation von chromatischer Substanz im Kern sekundär eine erhöhte Abgabe von Nucleolarsubstanzen an das Cytoplasma erfolgt, daß also mit anderen Worten eine Aktivierung der funktionellen Zellphase zustande kommt, deren Auswirkung auf den weiteren Ablauf des Lebens der Zelle zu untersuchen bleibt.

Nach einer früheren Mitteilung ${ }^{7}$ ist anzunehmen, daß bei den Zellen des MAC in der Kernsekretion solche Stoffe an das Cytoplasma abgegeben werden, welche die Zelle zum weiteren Ablauf ihrer primitiven vitalen Funktionen, insbesondere in der Phase der Teilung benötigt. Es ist aber auch zu überlegen, ob auf diese Weise die sog. "Teilungsstoffe" in das Cytoplasma gelangen, die nach $\mathrm{M} u ̈ h \mathrm{ld}$ orf ${ }^{13}$ zur ordnungsgemäßen Durchführung einer Plasmateilung hier vorhanden sein müssen. Jedenfalls kann eine Zellteilung erst dann einsetzen, wenn sich der DNSGehalt des Kernes verdoppelt hat und das Cytoplasma mit den für die weiteren Vorgänge notwendigen Substanzen versorgt ist. Der Funktions-Formwechsel bekommt dadurch bei den Zellen des MAC teilweise die Bedeutung eines Auslösungsvorgangs, der ablaufen muß, um die Zelle in den Zustand der Teilungsbereitschaft zu versetzen. Seine Aktivierung muß demgemäß, auch wenn sie nur, wie im vorliegenden Falle, eine Folge der vorhergehenden Kondensation chromatischer Substanz im Kern ist, mit dazu beitragen, die Teilungstendenz der Zellen zu erhöhen. Dahingehend ist auch der Befund von Pseudoamitosen in den Zellausstrichen nach Colchicin-Einwirkung sowie der beschriebene Kernzerfall in viele Kleinkerne $\mathrm{zu}$ deuten. Hier wie dort ist eine deutliche, wenn nicht gar gesteigerte Tendenz zur Teilung vorhanden. Vielleicht liegt sogar in dieser Steigerung die Ursache dafür, daß in der Pseudoamitose ein Teilungsversuch des Cytoplasmas ohne Mitbeteiligung des Kernes, und beim Kernzerfall

13 A. M ü h ld orf, Die Zellteilung als Plasmateilung, Springer 1951. 
eine Kernteilung ohne synchrone Teilung des Plasmakörpers durchgeführt wird. Daß aber auch im letzteren Falle im Cytoplasma eine Teilungsbereitschaft besteht, zeigt der auch zu beobachtende, scheinbar regellose, später erfolgende Plasmazerfall, der nach $\mathrm{M} \ddot{\mathrm{u} h l d}$ orf einen Gehalt des Cytoplasmas an Teilungsstoffen zur Voraussetzung hat.

Nachdem die Definition des Colchicins als Spindelgift mit ausgesprochen spindel-arretierender Wirkung allgemein anerkannt ist, erhebt sich die Frage, ob die hier geschilderten Veränderungen an Zellen des MAC als Folge einer spezifischen Colchicinwirkung aufzufassen sind. Wir möchten diese Frage verneinen mit dem Hinweis auf Befunde, die sich in ähnlicher Weise nach Einwirkungen von Unterkühlungen oder nach Behandlung mit schnellen Elektronen beobachten lassen ${ }^{14,15}$. In beiden Fällen zeigen sich ähnliche morphologische Erscheinungen, wie wir sie hier beschrieben haben. Sie sind zu deuten als Reaktion der MAC-Zellen auf allgemeine, unspezifische Schädigungen und die Reaktion

14 W. Homann u. D. Hofmann, Strahlenther. 83, 291 [1950]. selbst als eine Art biologischen Versuchs, eine „Phase schlechter Umweltbedingungen zu überwinden“.

Eine kurze Gegenüberstellung der geschilderten Befunde nach Einwirkung von Trypaflavin bzw. Colchicin auf Zellen des MAC zeigt, daß in den Versuchen zwei Typen von Einflußnahme auf den Funktions-Formwechsel erzielt wurden. Im Falle des spezifisch wirkenden Trypaflavins führen schwere Strukturänderungen innerhalb des Kernes zu einer völligen Unterbindung der Ausbildung eines Funktions-Formwechsels, das unspezifisch wirkende Colchicin hingegen löst eine Aktivierung der funktionellen Zellphase aus. Die Angriffspunkte liegen zeitlich in beiden Fällen vor der eigentlichen Funktionsphase der Zellen, wenngleich Colchicin möglicherweise auch in einen schon ablaufenden FunktionsFormwechsel eingreifen kann. Ob es möglich ist, mit chemischen Substanzen direkt in den FunktionsFormwechsel einzugreifen oder ob es noch andere Reaktionstypen des Funktions-Formwechsels gibt, muß weiteren Untersuchungen überlassen bleiben.

15 W. H o m a n n, Krebsarzt 9, 223 [1954].

\title{
Alternsgänge in der Großhirnrinde des Menschen
}

\author{
Von Rudolf Ehrenberg und Margarete Pöhl \\ Aus dem Physiologischen Institut der Universität Göttingen \\ (Z. Naturforschg. 9 b, 796-798 [1954]; eingegangen am 22. Oktober 1954)
}

\begin{abstract}
In der Großhirnrinde des Menschen wurde der Bindegewebsanteil sowie die Größe der Betzschen Riesenzellen bestimmt.

Beide Werte zeigen eine Zunahme mit dem Altern, doch ist der kontinuierliche Anstieg, zumal bei dem Bindegewebe, weniger ausgeprägt als an Herz und Leber. Die Breite der individuellen Schwankungen um einen Mittelwert ist wesentlich größer als bei den früher untersuchten Organen, sie nimmt mit dem Altern deutlich zu.
\end{abstract}

$I^{\prime}$ $\mathrm{n}$ einer früheren Arbeit ${ }^{1}$ wurden Untersuchungen mitgeteilt, die den Alternsgang der Bindegewebszunahme in Herz und Leber des Menschen zum Gegenstand hatten. Es hatte sich ein durch das ganze Leben kontinuierlich fortschreitender Anstieg des Bindegewebsanteils ergeben, der für beide Organe von gleicher Steilheit war. Von der Anschauung ausgehend, daß das Gehirn eine grundsätzlich andere Stellung im Lebensgeschehen einnimmt als die rein funktionalen Organe, daß es den eigentlichen Träger des Lebensablaufes, das Alternsorgan

1 R. Ehrenberg, H. G. Winnecken u. H. Bichricher, Z. Naturforschg. 9 b, 492 [1954]. schlechthin darstellt, daß also der Alternsgang hier als Wesensbestimmung erscheint, von dieser Anschauung ausgehend wandten wir uns nunmehr der Untersuchung von Alternsgängen in den Gehirnen menschlicher Leichen des Alters von 0-90 Jahre zu. Hier handelte es sich darum, nicht nur die Gewebsarten als solche in ihren Anteilen zu bestimmen, sondern an den Zellen selbst Alternsveränderungen zu untersuchen. Die sogenannten Alterspigmente der Ganglienzellen, deren frühzeitiges Auftreten und beträchtliches Zunehmen im höheren Alter seit langem bekannt ist, behielten wir späteren Untersuchungen vor, die nachstehenden Ergebnisse betreffen 